Running head: EXPOSURE TO UNTRUSTWORTHY WEBSITES IN 2020

Exposure to Untrustworthy Websites in the 2020 US Election

\author{
Authors: \\ Ryan C. Moore* \\ (Corresponding Author) \\ PhD Candidate \\ Department of Communication \\ Stanford University \\ Stanford, CA, USA \\ https://orcid.org/0000-0002-5669-721X \\ rymoore@stanford.edu \\ Ross Dahlke \\ PhD Candidate \\ Department of Communication \\ Stanford University \\ Stanford, CA, USA \\ https://orcid.org/0000-0002-5179-2525 \\ rdahlke@stanford.edu \\ Jeffrey T. Hancock \\ Harry and Norman Chandler Professor of Communication \\ Department of Communication \\ Stanford University \\ Stanford, CA, USA \\ https://orcid.org/0000-0001-5367-2677 \\ hancockj@stanford.edu
}




\begin{abstract}
Research using large-scale data on individuals' internet use has provided vital information about the scope and nature of exposure to misinformation online. However, most prior work relies on data collected during the 2016 US election. This paper examines exposure to untrustworthy websites during the 2020 US election using 7.5 million website visits from 1,151 American adults. We find that $26.2 \%$ of Americans were exposed to untrustworthy websites in 2020 , down from $44.3 \%$ in 2016 (as calculated by Guess et al. [2020]). Older adults and conservatives continued to be the most exposed in 2020 as in 2016 , albeit at lower rates. The role of online platforms in exposing people to untrustworthy websites changed, with Facebook playing a smaller role in 2020 than 2016. Our findings do not minimize misinformation as a key social problem but instead highlight important changes in its consumption, suggesting directions for future research and practice.
\end{abstract}

Keywords: misinformation, 2020 U.S. election, web browsing, digital trace data 


\section{Exposure to Untrustworthy Websites in the 2020 US Election}

Both concern over and research on misinformation have exploded in recent years (Weeks \& Gil de Zúñiga, 2019). In an effort to better understand and prevent misinformation's spread, much extant research documents exposure to misinformation online using large-scale data on individuals' internet use. This work finds that relatively few people are exposed to misinformation online, or at least fewer than many initially expected (Allcott \& Gentzkow, 2017; Allen et al., 2020; Grinberg et al., 2019; Guess et al., 2019, 2020; Weeks et al., 2021). Exposure is also highly concentrated (Grinberg et al., 2019; Guess et al., 2019, 2020; Nelson \& Taneja, 2018). For example, Grinberg et al. (2019) found that $1 \%$ of Twitter users were exposed to $80 \%$ of the fake news on Twitter during the 2016 election. Relatedly, certain individuals are significantly more likely to be exposed to misinformation online than other groups. For example, during the 2016 election, people 65 and older were twice as likely to be exposed to fake news on Twitter and seven times more likely to share fake news on Facebook than 18-29 year-olds (Grinberg et al., 2019; Guess et al., 2019).

Many studies investigating exposure to online misinformation have leveraged data collected during the 2016 US election. This pattern may be because concern over fake news rose during the 2016 election ( $\mathrm{Li} \& \mathrm{Su}, 2020$ ). However, a consequence of focusing on the 2016 election is that we have little insight into how exposure to misinformation online has changed since then. This limitation is important given the myriad ways the digital (mis)information ecosystem has changed (e.g., new platforms, new misinformation-generating world events, new modalities to disseminate misinformation; Ball \& Maxmen, 2020; Lytvynenko, 2020). Furthermore, in the wake of 2016 , online platforms such as Facebook have taken steps to mitigate their reputation as a purveyor of misinformation (Lyons, 2018).

While most prior research focuses on 2016 , some work has examined changes in exposure over time. For example, Guess, Lyons, et al. (2019) found a decline in the number of 
Americans exposed to fake news websites from 2016 to 2018. By contrast, Allen et al. (2020) found that exposure to fake news was "generally stable" from 2016 through the end of 2018. Discrepancies between these two findings may partly be explained by the authors using different lists to identify visits to fake news websites in their data. Allcott et al. (2019) also examined changes in exposure over time, finding that engagement with fake news on Facebook declined from 2016 through 2018 while engagement with fake news on Twitter rose over the same period.

Given changes in world events, the digital media landscape, and a significant societal effort to combat misinformation, a vital question remains as to how misinformation exposure during the 2020 US presidential election compared to during the 2016 election, which served as the context for much of the existing research. In this paper, we investigate exposure to online misinformation during the 2020 election in a way that allows us to compare exposure in 2020 to 2016 directly. To do this, we collected web browsing data (i.e., URLs visited) from a nationally representative sample of American adults $(N=1,151)$ during the lead-up to the 2020 election (total number of website visits $=7.5$ million). We identify visits to misinformation websites in participants' web browsing using a database of websites known to repeatedly publish misinformation. To produce estimates of misinformation exposure directly comparable to those from 2016, we adopted the analytical approach of Guess et al. (2020), who studied exposure to misinformation during the lead-up to the 2016 election using web browsing data (see the Methods section at the end of the paper for full methodological details). While we stuck closely to the data sources and analytical techniques used by Guess et al. (2020), we made key updates that ensured our analysis was up-to-date for the media environment in 2020 , such as expanding the database of misinformation websites from 490 sites to 1,796 sites.

What might we expect about exposure to online misinformation in 2020 ? On the one hand, there are reasons to believe that exposure in 2020 was greater than in 2016 . The 2020 election was marked by a confluence of major national and global events that generated a 
considerable amount of misinformation to which people could have been exposed (e.g., the rise of the COVID-19 pandemic, claims that the 2020 presidential election was fraudulent; Lytvynenko, 2020). Furthermore, the infrastructure available to disinformation campaigns and purveyors for disseminating and spreading false information across platforms has improved considerably in recent years (Alaphilippe, 2021; Wilson \& Starbird, 2021). On the other hand, there are also reasons to believe that exposure in 2020 was lesser than in 2016 . Since the 2016 election, journalists and online platforms have taken several steps to curb the spread of misinformation. For example, social media platforms such as Facebook and Twitter have begun labeling and flagging untrustworthy content on their platforms (Lyons, 2018; Newton, 2020; Roth \& Achuthan, 2020), and journalists have intensified their efforts around accountability and transparency (Vu \& Saldaña, 2021). Furthermore, interventions and educational resources for helping the public identify online misinformation that have proliferated since 2016 may have improved internet users' ability to avoid misinformation (Fazio, 2020; Kozyreva et al., 2020). Finally, the few studies that have examined misinformation exposure post-2016 have identified a decline or no change in exposure (Allen et al., 2020; Guess, Lyons, et al., 2019).

Understanding exposure to online misinformation in 2020 is important for several reasons. First, it represents an effort to see whether the inferences drawn about the scope and nature of misinformation exposure from 2016 are valid in 2020, helping to enhance the "temporal validity" of the knowledge base on online misinformation exposure (for a greater discussion of the concept and utility of temporal validity in the context of social media research, see Munger, 2019, 2022). Second, it is relevant to the design of interventions to build resilience to misinformation. Understanding who is exposed to online misinformation -- and when and how they are exposed -- may help researchers identify how to make the content of interventions best suited for different types of users, and understanding the nature of misinformation exposure can inform policymaking efforts to mitigate misinformation (e.g., requiring social media platforms to implement certain flagging or fact-checking practices). Finally, updates about misinformation 
exposure can inform public discourse around misinformation itself. Since 2016, misinformation has been a significant topic in communication from journalists, elites, scientists, and the general public (Li \& Su, 2020), with implications for how the public trusts information sources (Jungherr \& Rauchfleisch, 2022). Indeed, exposure to elite discourse about misinformation has been shown to reduce people's trust in traditional news media (Van Duyn \& Collier, 2019). If it is true that the vast majority of people's news diet is composed of reliable news (Allen et al., 2020), then the heightened discourse around misinformation could erode trust in reliable news.

Overall, our analysis reveals that exposure to untrustworthy websites during the 2020 election was significantly lower than Guess et al. (2020) determined during the 2016 election. Not only was the proportion of total individuals exposed to untrustworthy websites lower, but the average number of exposures among the exposed and the average amount of time spent on each site were also lower. While we also found that the groups who were more likely to be exposed to untrustworthy websites in 2016 -- older adults, conservatives -- were still more likely in 2020, levels of exposure for these groups were lower than in 2016 . We found these patterns despite using a database of untrustworthy websites over three times the size used in Guess et al. (2020), meaning that even with a considerably larger capacity to identify misinformation in people's web browsing data, we still found less. Finally, we found changes in how people come to be exposed to untrustworthy websites, namely that people were less likely to arrive at them via Facebook and webmail in 2020 than in 2016.

\section{Results}

\section{Total Consumption of Untrustworthy Websites in 2020}

During the 2016 election, Guess et al. (2020) found that $44.3 \%(95 \% \mathrm{Cl}=40.8-47.7 \%)$ of Americans aged 18 or older were exposed to at least one untrustworthy website. By contrast, we find that during the 2020 election, only $26.2 \%(95 \% \mathrm{Cl}=22.5-29.8 \%)$ of Americans were exposed to at least one untrustworthy website. This decrease represents a significant reduction $(\mathrm{t}(2,2493.6)=-11.12, p<.001$, Cohen's $d=.39,95 \% \mathrm{Cl}=[-19.8,-16.5])$ in the percentage of 
Americans exposed to untrustworthy websites from 2016 to 2020 (see Supplementary Materials for additional estimates of 2020 exposure using NewsGuard's 0-100 "trust score").

While a smaller percentage of Americans appeared to be exposed to untrustworthy websites in 2020 compared to 2016 , one possibility is that those who were exposed to untrustworthy websites in 2020 were exposed at greater rates than in 2016 . However, our data suggest otherwise. First, the average number of untrustworthy website visits among those who visited at least one untrustworthy website in 2020 (22.8 visits; 95\% Cl $=15.2-30.4)$ was significantly lower than the average number in $2016(32.1$ visits; $95 \% \mathrm{Cl}=26.3-37.9)(\mathrm{t}(2,638)$ $=-2.06, p=.04, d=.12,95 \% \mathrm{Cl}=[-13.8,-4.8])$. Second, the average length of time spent on untrustworthy websites was shorter in 2020 (38.6 seconds; $95 \% \mathrm{Cl}=34.5-42.7$ s) than in 2016 (64.2 seconds; Guess et al. [2020] did not quantify uncertainty for this quantity).

Comparing visits to untrustworthy websites in 2020 and 2016, we find that overall consumption of untrustworthy sites was lower in 2020 than in 2016 (see also the Supplementary Materials for an analysis of untrustworthy website consumption from 2016 to 2020 using available estimates from other papers). Fewer Americans were exposed to untrustworthy websites in the lead-up to the 2020 election, and those who were exposed visited fewer and were less engaged with the untrustworthy sites they visited. Next, we examine how the types of people more likely to visit untrustworthy websites in 2016 compared to those who were more likely to visit them in 2020 .

\section{Who Consumed Untrustworthy Websites in 2020?}

In 2016, Guess et al. (2020) found that supporters of then-presidential candidate Donald Trump were significantly more likely to visit untrustworthy websites than supporters of his opponent Hillary Clinton, with 59.5\% (95\% Cl = 54.5-64.5\%) of Trump supporters visiting at least one untrustworthy website and $37.1 \%(95 \% \mathrm{Cl}=31.9-42.2 \%)$ of Clinton supporters visiting at least one untrustworthy website. We find a similar difference between Donald Trump and Joe Biden supporters in 2020. 36.2\% (95\% Cl = 29.7-42.6\%) of Trump supporters visited at least 
one untrustworthy website in 2020 while $17.8 \%(95 \% \mathrm{Cl}=13.7-22.0 \%)$ of Biden supporters visited at least one untrustworthy website. However, for both Trump and Clinton/Biden supporters, the percentages of supporters exposed in 2020 were significantly lower than the percentages of supporters exposed in $2016\left(\mathrm{t}_{\text {Trump }}(2,818.8)=-8.20, p<.001, d=.48,95 \% \mathrm{Cl}=\right.$ $\left.[-26.2,-20.5] ; t_{C l i n t o n / B i d e n}(2,1654.8)=-9.53, p<.001, d=.44,95 \% \mathrm{Cl}=[-21.2,-17.2]\right)$.

Guess et al. (2020) further examined the association between political leaning and consumption of untrustworthy websites by classifying users based on the ideological slant of their news diets and examining exposure to untrustworthy websites among those with more liberal or conservative news diets. Once they estimated the average ideological position of participants' news diets (using estimates of the ideological leaning of hard news websites produced by Bakshy et al. [2015]), they split their participants into news slant deciles ranging from most liberal to most conservative. We implemented this same classification strategy on our sample to examine how exposure to untrustworthy websites across people with news diets with different ideological slants in 2020 compares to 2016.

This comparison is presented in Figure 1 (Table S7 contains the estimates comprising Figure 1). In 2016, there was a skewed V-shape in the relationship between media slant decile and the percentage of people exposed to untrustworthy websites. In 2016 , roughly half of the people in the most liberal media slant deciles were exposed to untrustworthy websites. That percentage decreases moving toward more moderate media diets but then increases among the most conservative diets, with $85.2 \%(95 \% \mathrm{Cl}=76.7-93.9 \%)$ in the most conservative media diet being exposed to untrustworthy websites. In 2020, the pattern resembles something closer to a left-skewed distribution, where the percentage of people exposed to untrustworthy websites is relatively constant, moving from the most liberal to more moderate media diets but then again increases dramatically among the most conservative diets. Due to these different patterns, the percentage of people exposed to untrustworthy websites in more liberal media diets in 2020 was considerably lower than in those diets in 2016. Among the most conservative media diets, 
the percentage of those exposed to untrustworthy sites was similar across both years. Put another way, these results indicate that decreases in untrustworthy website exposure from 2016 to 2020 were concentrated mainly among those with liberal media diets.

\section{[FIGURE 1 HERE]}

Age also played an important role in exposure to untrustworthy websites during the 2016 election, with older individuals generally being more likely to visit untrustworthy sites. In 2016, those 65 and older were 1.6 times more likely to visit untrustworthy websites than adults under 30. During the 2020 election, it appears that the positive association between age and likelihood of exposure to untrustworthy websites persisted, with those 65 and older being 2.1 times more likely to visit untrustworthy websites than those under 30 . However, all age groups in 2020 were less likely to be exposed to untrustworthy websites than in 2016 (see Figure 2). For instance, while $56.2 \%(95 \% \mathrm{Cl}=49.7-62.7 \%)$ of people 65 and older were exposed to untrustworthy websites in 2016 , only $37.4 \%(95 \% \mathrm{Cl}=29.6-45.2 \%)$ of people 65 and older were exposed in 2020.

[FIGURE 2 HERE]

To examine the association between a variety of individual differences and exposure to untrustworthy websites, we follow the strategy used by Guess et al. (2020) and estimate a linear probability model for the 2020 election period. In this model, the dependent variable is a binary variable indicating whether an individual was (1) or was not (0) exposed to at least one untrustworthy website during the data collection period. The independent variables include presidential candidate support, political knowledge, political interest, level of education, gender, race, and age. The models for 2016 and 2020 are presented in Table 1. Across both 2016 and 2020, many of the same demographic variables were significantly associated with exposure to untrustworthy websites (substantively similar results are obtained when probit models are estimated, see Table S1). Specifically, being a Trump supporter, possessing greater political knowledge, being more interested in politics, and being 65 years of age or older were 
associated with a significantly greater likelihood of being exposed to at least one untrustworthy website in both 2016 and 2020, while identifying as non-white was associated with a significantly lower likelihood of being exposed to at least one untrustworthy website in both years.

\section{[TABLE 1 HERE]}

Taken together, these findings suggest that the groups who were more likely to be exposed to untrustworthy websites in 2016 were largely the same groups more likely to be exposed in 2020. However, the likelihood of exposure in 2020 for these groups still appears to be lower than their rates in 2016 . That is, while trends in who was exposed to misinformation in 2016 seem to persist in 2020 , the amount all groups were exposed to was lower across the board.

\section{How Were People Exposed to Untrustworthy Websites in 2020?}

Guess et al. (2020) examined how individuals came to visit untrustworthy websites during the 2016 election by analyzing "referrers" to untrustworthy websites, or the sites that were within 30 seconds and one of the three previous sites before an untrustworthy website exposure. We repeated this analysis of referrers to untrustworthy websites for the 2020 election. The comparison in referrers between the 2016 and 2020 elections is presented in Figure 3.

In 2016, Facebook was a prominent referrer to untrustworthy websites, with $15.1 \%$ of visits to untrustworthy websites being referred by Facebook. In 2020 , however, only $5.6 \%$ of visits to untrustworthy sites were referred by Facebook, a significant reduction $\left(X^{2}(1)=573.4, p\right.$ $<.001, \varphi=.10,95 \% \mathrm{Cl}=[-10.7,-8.9])$. This suggests a potential decline in Facebook's role in directing people to untrustworthy websites. Conversely, the share of untrustworthy websites referred by Google rose from 2016 to 2020, with 3.3\% of visits to untrustworthy sites referred by Google in 2016 and $6.2 \%$ of visits referred by Google in $2020\left(X^{2}(1)=181.2, p<.001, \varphi=.05\right.$, $95 \% \mathrm{Cl}=[2.4,3.4])$. However, when looking at the average number of referrals to untrustworthy websites per exposed person, we see that while Facebook's average number of untrustworthy 
website referrals per person fell from 6.4 in 2016 to 1.4 in 2020, Google's average number of referrals per person stayed relatively constant, going from 1.4 in 2016 to 1.52020 , suggesting caution in interpreting the proportional results indicating that Google's role in referring people to untrustworthy websites increased from 2016 to 2020. Furthermore, Google referred a greater proportion of visits to credible, hard news websites in $2020(8.5 \%)$ than in $2016(6.2 \%)\left(X^{2}(1)=\right.$ 2428.7, $p<.001, \varphi=.20,95 \% \mathrm{Cl}=[2.3,2.4])$. In addition to Facebook, webmail (e.g., Gmail) also played a smaller role in referring people to untrustworthy websites in 2020 compared to in 2016 , both in proportional $(9.5 \%$ of visits to untrustworthy websites referred by webmail in 2016 vs. $5.7 \%$ of visits in $\left.2020 ; \mathrm{X}^{2}(1)=130.4, p<.001, \varphi=.10,95 \% \mathrm{Cl}=[-10.1,-8.9]\right)$ and averageper-person (an average of 4.0 referrals in 2016 vs. an average of 1.4 referrals in 2020) terms.

\section{[FIGURE 3 HERE]}

\section{Discussion}

Our goal in this paper was to examine how exposure to misinformation online during the 2020 election compared to exposure during the 2016 election. By adopting the analytical approach of Guess et al. (2020), who examined exposure to untrustworthy websites during the 2016 election, we assessed exposure to untrustworthy websites among a nationally representative sample during the 2020 election and compared it directly to 2016 exposure. Our web browsing data containing 7.5 million URLs represent observations of $N=1,151$ Americans' real-world media usage during the course of real-world political events. Our analysis does not rely on self-reported media exposure measures, which tend to be inaccurate compared to passively tracked behavioral measures of news exposure (Guess, 2015; Konitzer et al., 2021).

Overall, we found that a significantly smaller percentage of Americans were exposed to untrustworthy websites in 2020 (26.2\%) compared to 2016 (44.3\%). We found this decrease despite using a database of untrustworthy sites over three times the size of the database used in Guess et al. (2020) to identify visits to untrustworthy websites in our participants' web browsing behavior, which increased our capacity to detect visits to untrustworthy websites. This 
decline runs contrary to expectations that the run-up to the 2020 election would lead to record numbers of people being exposed to misinformation (e.g., Lytvynenko, 2020; but also for reasons to predict lower misinformation exposure in 2020 see Kozyreva et al., 2020; Newton, 2020; Vu \& Saldaña, 2021). We also observed that those who did visit untrustworthy websites in 2020 tended to visit fewer untrustworthy sites overall and spent less time on average on each site than in 2016.

In 2016, certain groups were more likely than others to visit untrustworthy websites. Older adults were found to be more likely to visit untrustworthy sites than younger adults, supporters of Donald Trump were more likely than supporters of Hillary Clinton, and those with more ideologically extreme media diets were more likely than those with ideologically moderate media diets. We found that these groups were still more likely to visit untrustworthy websites in 2020 (in 2020, Joe Biden supporters took the place of Hillary Clinton supporters). However, the groups' levels of exposure were significantly lower in 2020 than in 2016 . While it is encouraging that the likelihood of encountering untrustworthy websites appears to be declining over time for older adults, Trump supporters, and those with ideologically extreme media diets, the data reaffirm the need to support these groups in terms of future research and the provision of resources to build resilience to misinformation.

Finally, we found differences in how people came to visit untrustworthy websites in 2020 compared to in 2016. Specifically, Facebook and webmail referred significantly fewer visits to untrustworthy websites in 2020 than in 2016 . While further research is needed to elucidate these platforms' changing role in propagating misinformation (see below), our findings suggest that Facebook and webmail may have played a smaller role in directing people to misinformation on the web in the 2020 election compared to in 2016.

While our data suggest that exposure to untrustworthy websites declined at the population level, our results should not be interpreted as indicating that misinformation is somehow less of a problem than it was previously. While exposure was lower in 2020 than in 
2016, many people were still exposed to untrustworthy sites. Extrapolating our results suggests that nearly 68 million Americans made a total of 1.5 billion visits to untrustworthy sites during the 2020 election. Furthermore, even if a smaller percentage of Americans were exposed to misinformation online in 2020 , those exposures could have played a larger role in radicalization or influencing participation in acts of political violence (e.g., the January 6, 2021 insurrection). Altogether, exposure to fewer people can still have serious consequences. While our data and approach are limited in their ability to speak to the consequences of exposure, it will be essential for future scholarship on misinformation to consider both exposure to misinformation and the effect of that exposure at the population and individual levels.

We make several key contributions to the misinformation literature. First, our study demonstrates the value of reapplying the same analytical approach of prior work to examine changes in a mediated process (e.g., exposure to untrustworthy websites) longitudinally. By collecting the same data (URLs visited during web browsing) from the same source (YouGov Pulse) among the same population (nationally representative sample of American adults) for a similar period of time around the US Presidential election as Guess et al. (2020), we were able to make direct comparisons of exposure to untrustworthy websites between 2016 and 2020 . The apples-to-apples comparisons afforded by this approach allowed us to precisely examine how the patterns in untrustworthy website exposure identified in 2016 changed in 2020. In addition, we incorporated improvements to Guess et al. (2020)'s approach into our analysis that accounted for differences between the 2016 and 2020 media environments. For example, we introduced NewsGuard's database of sites that repeatedly publish false content as an additional source to identify visits to untrustworthy websites in people's web browsing. One reason the introduction of NewsGuard was important is that the untrustworthy website databases used by Guess et al. (2020) were primarily based on websites circulating during the 2016 election, but fake news websites are often ephemeral (i.e., the domains go defunct after short periods of time; Lim et al., 2019). NewsGuard's database (which is updated weekly) allowed us to have 
more confidence that our database of untrustworthy sites was sufficiently up-to-date to match changes in the fake news ecosystem that have occurred since 2016.

Second, our findings indicate that the same groups who were more likely to visit untrustworthy websites in 2016 were largely the same more likely to do so in 2020 . The persistence of these trends highlights the importance of examining why populations like older adults appear to be more susceptible to online misinformation and how they can be supported through interventions and other resources to build resilience to misinformation (for a review, see Brashier \& Schacter, 2020; Moore \& Hancock, 2022). Our updated findings, which reveal that approximately one-third of the older adults in our sample were exposed to untrustworthy websites, make clear that it is important to continue studying the factors responsible for older adults' vulnerability.

In addition to this pattern among older adults, the 2016 pattern of conservatives being more likely than liberals to visit untrustworthy sites persisted in 2020. Research has begun to identify why conservative individuals appear more likely to engage with misinformation online (Freelon et al., 2020). One reason might be that the supply of misinformation is greater on the ideological right than on the left (Benkler et al., 2018; Garrett \& Bond, 2021). Relatedly, it could be that more ideologically conservative media diets are more likely to expose users to misinformation via features like algorithmic curation and community structures relative to liberal media diets. The left may also be more likely to circulate misinformation via modalities other than website links (e.g., social media posts, memes and other image-based formats) that are more difficult to detect using URL-based web tracking methods. Our results suggest an ongoing need for future work to investigate these potential causes.

Third, we provide evidence that Americans' visits to untrustworthy websites were less likely to be referred by Facebook in 2020 than 2016. This finding is significant given the amount of scrutiny from members of Congress and the American public towards platforms', such as Facebook, roles in the proliferation of misinformation (Auxier, 2020; Wakefield, 2021). Future 
work should endeavor to understand better why Facebook's role in referring people to untrustworthy websites appears to be shrinking. Does it indicate the efficacy with which Facebook is implementing programs and policies to label or flag untrustworthy content? Or might it suggest a more fundamental behavioral change in how people use Facebook and other social media platforms? For instance, people may be less likely to click links to external websites now than in the past, preferring to stay on the platform. Unfortunately, as the recent Facebook Files controversy illustrates, without these platforms sharing data and becoming more transparent, it is difficult to understand these changes in referral patterns (The Wall Street Journal, 2021). We add our voices to those calling for increased data sharing and independent research of platform data (Edelson \& McCoy, 2021; Persily, 2021).

Finally, our findings can inform policymaking and public discourse around misinformation. Our findings reaffirm that certain groups (older adults and conservatives) are more likely to encounter misinformation, suggesting a need for more focused and directed policy initiatives centering on groups with the greatest need for support in dealing with misinformation. Moreover, our findings join others suggesting that the attention and discussion that the media, politicians, and the public devote to fake news may be disproportionate to the extent that people are actually exposed to it (Jungherr \& Rauchfleisch, 2022; Weeks et al., 2021). Given evidence that a) the overwhelming majority of news consumed by the population is not misinformation (Allen et al., 2020), and b) that exposure to discourse around fake news can erode individuals' trust in news media (Van Duyn \& Collier, 2019), we may need to consider the nature of the attention society pays to the problem of misinformation relative to other ongoing national and international crises. For example, the emphasis on misinformation present in the media and political discourse since 2016 may be partly to blame for the ongoing erosion of trust in media institutions occurring in the United States and worldwide (Edelman, 2021). Our findings call for the need for more research but also more grounded communication of that research to appropriately contextualize this phenomenon. 
Of course, our study should be interpreted in light of its limitations. The most noteworthy limitations relate to our URL logging methodology to estimate untrustworthy website exposure. First, untrustworthy websites were operationalized at the domain level. That is, in our data, untrustworthy websites were considered web domains that NewsGuard rated as repeatedly hosting false information (or imported from the database used by Guess et al. [2020]) (e.g., www.obamawatcher.com), rather than specific webpages or articles (e.g., www.obamawatcher.com/2020/03/michelles-fake-degrees). This operationalization is largely because most URLs captured by YouGov only contain domains and not full URLs to protect participant privacy. While many studies examining exposure to misinformation have taken this approach (Allen et al., 2020), there is undoubtedly misinformation hosted on domains that do not repeatedly publish false information but may occasionally publish false information. Domainlevel measurements of exposure do not capture these specific instances of misinformation.

A second limitation of URL-based browsing data is that it only identifies content that leads to a URL being produced (Ellison et al., 2020). Crucially, this limitation means that for web pages that display content dynamically while maintaining a static URL, we only know that a person visited that static URL but not information about any of the content they saw while on that static URL. Take, for example, Facebook's Feed. When a user visits www.facebook.com and is presented with their Feed, that user can scroll through their Feed and that does not result in the active URL in their web browser, www.facebook.com, changing. Thus, while an individual may be exposed to a variety of (mis)information while scrolling their Feed (either in posts generated or links shared by others), we only observe instances in which individuals actually click on an external link which takes them away from their Feed to a new website.

Additionally, participants collected these URL data by installing plugins in their web browsers. Thus, we only capture individuals' behaviors within web browsers. Individuals' online behaviors outside of web browsers, such as through apps, do not appear in our dataset. This nuance may be especially relevant for mobile internet use, which is more likely to occur via 
mobile apps than mobile web browsers (Wurmser, 2019). Indeed, among our participants, we found that more individuals were exposed to an untrustworthy website on desktop/laptop computers $(34.2 \% ; 95 \% \mathrm{Cl}=29.4-39.1 \%)$ than on smartphones and tablets $(13 \% ; 95 \% \mathrm{Cl}=$ $8.9-17.2 \%)$. As the consumption of news and political information increasingly occurs on mobile devices, it becomes all the more important for researchers to invest in tools and methods that allow for the collection of mobile browsing and app-use data to increase the validity of inferences about online news exposure (González-Bailón \& Xenos, 2022).

It is important to note that these limitations affect both our 2020 data collection and Guess et al. (2020)'s 2016 data collection. Nevertheless, it is crucial for future research on misinformation exposure to contend with the limitations of web browsing data. Only social media companies ultimately possess the data on user behavior that could most accurately shed light on why their role in referring individuals to untrustworthy websites appears to be decreasing over time. Unfortunately, social media platforms rarely share data with misinformation researchers (Pasquetto \& Swire-Thompson, 2020). While initiatives such as Social Science One have attempted to grant scholars access to data from social media platforms like Facebook (King \& Persily, 2020), getting academic researchers access to platform data has proven difficult (Edelson \& McCoy, 2021; Persily, 2021). The limitations of our study and those that other misinformation research face highlight the importance of social media platforms working with academic and other third-party researchers to better understand the complex dynamics of exposure to and engagement with (mis)information on their platforms.

That said, other current data collection approaches could help fill in the gaps in online behavior missed by the URL-logging method. For example, Screenomics captures screenshots from individuals' devices to understand their moment-by-moment smartphone usage, including the use of apps and information contained within system notifications (Reeves et al., 2019, 2020). The Screenomics method has been used to study exposure to political information (Muise, 2022). Future research on exposure to online misinformation should triangulate across 
several data sources to gain a more complete portrait of individuals' online media use and the role that misinformation plays in it.

Our findings indicate a relatively across-the-board decline in exposure to untrustworthy websites from 2016 to 2020 , but why does this decline occur? We offer a few candidate explanations, but future work and additional data will be needed to test them more directly.

First, exposure to misinformation could have been more likely to be displaced in 2020 than in 2016 to other locations outside the web browser, such as text messaging or emergent social media apps like WhatsApp and TikTok. Indeed, people increasingly report getting their news regularly via WhatsApp (Newman et al., 2020) and social media platforms like Reddit and TikTok (Walker \& Matsa, 2021), and there is concern about the spread of misinformation on these platforms (Delcker et al., 2020; Zadrozny, 2021).

Second, the time frame of data collection used by Guess et al. (2020) and adopted by us (four weeks prior to Election Day and one week after Election Day) may have examined different misinformation dynamics around the 2020 and 2016 elections. Specifically, the 2020 election was marked by a post-Election Day period in which sitting president Donald Trump made a series of claims about election fraud that caused him to receive less votes than his opponent Joe Biden, culminating in the announcement that Joe Biden was the winner of the election on November 7, which Donald Trump refused to concede. During this time, much misinformation about election fraud circulated online (Frenkel, 2020). Comparatively, the aftermath of the 2016 election may not have been as rife with online misinformation as the aftermath of the 2020 election. However, because Guess et al.'s (2020) (and thus our) data collection period included more time before the election than after, we may have missed some of the misinformation relevant to the 2020 election outcome that was not present in 2016 . This demonstrates that when comparing the effects of events on media consumption behaviors, even similar events (e.g., presidential elections) may feature different dynamics at different points in time. 
Third, as we pointed out in the Limitations section, URL-tracking methods only log instances in which a URL is actually clicked and visited. Visits to untrustworthy websites may have decreased from 2016 to 2020 because people were increasingly exposed to untrustworthy website content within dynamic URLs, for example, scrolling through the Facebook Feed or Twitter Timeline. Indeed, evidence suggests that relatively few people click on news links posted on social media websites (Ju et al., 2014; Mitchell \& Jurkowitz, 2014) yet can be influenced by the information in headlines (Rapp \& Salovich, 2018). This propensity to stay on the platform over clicking to visit external websites also may be increasing over time (Geeng et al., 2020). Such a change in user behavior could also help explain why we found that Facebook played a smaller role in referring people to untrustworthy websites in 2020 than in 2016.

There are two explanations for the decline in exposure to untrustworthy websites from 2016 to 2020 that we can address with our data. First, it is possible that the decline in untrustworthy website consumption from 2016 to 2020 reflected a broader decline in online news consumption overall, both credible and untrustworthy. Using the news websites contained in Bakshy et al. (2015) and those rated by NewsGuard as not repeatedly publishing false content, we find that Americans' exposure to hard news websites in 2016 did not significantly differ from exposure to hard news sites in 2020. The number of Americans exposed to at least one hard news site, the average number of hard news sites accessed, and the average amount of time spent on hard news sites were similar in 2016 and 2020 (see Supplementary Materials). These results suggest that untrustworthy news exposure uniquely declined from 2016 to 2020 while exposure to trustworthy news remained constant.

Another possibility is that consumption of untrustworthy websites fell from 2016 to 2020 because individuals' use of online fact-checking resources increased during that time. Exposure to fact-checking resources can reduce people's engagement with misinformation online (Aslett et al., 2022; Guess, 2015; Kauk et al., 2021; Nekmat, 2020; Vijaykumar et al., 2021), and using fact-checks is a strategy commonly taught in effective digital media literacy interventions (e.g., 
Brodsky et al., 2021; Wineburg et al., 2022). During the 2016 election, Guess et al. (2020) found that $25.3 \%(95 \% \mathrm{Cl}=22.5-28.2 \%)$ of Americans visited a fact-checking website. During the 2020 election, we estimate that $13.1 \%(95 \% \mathrm{Cl}=11.2-15.2 \%)$ of Americans visited a factchecking site, around half as many as in 2016 (see Figure S4). In 2016, approximately 42\% of those exposed to at least one untrustworthy website were exposed to at least one fact-checking website. In 2020 , this number fell to less than $30 \%$. In sum, it does not appear that the use of fact-checking websites increased from 2016 to 2020 , both among those exposed to misinformation and the population as a whole, casting doubt on the idea that exposure to misinformation decreased from 2016 to 2020 because the use of fact-checking resources increased during that time.

In this paper, we provide evidence that exposure to untrustworthy websites decreased from 2016 to 2020 . More research is needed to understand the factors explaining this change, but our results represent an important update to our understanding of exposure to online misinformation. The groups most likely to be exposed in 2016 are much the same groups who were more likely exposed in 2020 , justifying a more focused approach to research on and support for those groups in dealing with misinformation. While one could interpret our findings as evidence that the problem of online misinformation is improving in some way, they could also be interpreted as evidence that the nature of the problem is changing. Our work provides some initial insights into where researchers can start looking to understand the changing dynamics of online misinformation exposure. 


\section{Methods}

\section{Review of Guess et al. (2020) Methods}

Guess et al. (2020) is a widely-cited study examining exposure to online misinformation during the 2016 US election. The authors collected data from a national sample of American adults $(N=2,525)$ during the lead-up to the 2016 election (October 7 - November 14, 2016; Election Day was November 8, 2016) using the survey company YouGov. Specifically, they collected two types of data from participants: (1) web browsing data collected via YouGov's Pulse browser plugin, which is installed by survey respondents on their web browsers and collects all URLs visited as they surf the internet and (2) demographic information collected via surveys. They then combined these data with a list of 490 web domains that "frequently publish factually dubious or untrustworthy content" (Guess et al., 2020, p. 479) in order to identify visits to untrustworthy websites in their participants' web browsing data.

Similar to most research on misinformation exposure (Allen et al., 2020), Guess et al. take a domain-level approach to identifying misinformation. This approach means that rather than identifying specific webpages which contain misinformation (e.g., www.obamawatcher.com/2020/03/michelles-fake-degrees), Guess et al. (2020) rely on a list of domains that are known to frequently publish untrustworthy content (e.g., www.obamawatcher.com).

By matching their list of untrustworthy websites against their participants' web browsing data, Guess et al. found that $44.3 \%$ of their nationally representative sample were exposed to at least one untrustworthy website in the lead-up to the 2016 election. They also found that supporters of Donald Trump, those with more conservative media diets, and those over the age of 65 were more likely to visit untrustworthy websites. Finally, they found that exposures to untrustworthy websites frequently occurred via Facebook.

Our goal was to adopt the analytic approach of Guess et al. (2020) to analyze data collected via the same source (YouGov Pulse) during the same period (four weeks prior to 
Election Day and one week after Election Day) around the 2020 election in order to compare exposure to untrustworthy websites during the 2020 election to exposure during the 2016 election. We do not repeat every analysis found in Guess et al. (2020) in this paper, although several additional analyses can be found in our Supplementary Materials. Similar to Guess et al. (2020), our data distributions were assumed to be normal but this was not formally tested. The central advantage of adopting Guess et al.'s analytical approach is that we can make relatively direct comparisons between our 2020 estimates and their 2016 estimates.

\section{Participants}

To measure exposure to untrustworthy websites during the 2020 election, we passively gathered web browsing data (across smartphones, laptops, and desktop computers) from 1,151 Americans using YouGov's Pulse browser plugin from October 2, 2020 to November 9, 2020 (Election Day was November 3, 2020). These participants also completed an online survey that complemented their web browsing data. All participants consented to the terms of the research and were compensated by YouGov for their participation. Of these participants, $58 \%(n=670)$ supported Joe Biden in the 2020 election while $36 \%(n=419)$ supported Donald Trump. Twenty-nine percent $(n=329)$ were aged $65+, 48 \%(n=554)$ were $45-64,15 \%(n=171)$ were $30-44$, and $8 \%(n=97)$ were under 30 . Forty-five percent $(n=522)$ reported identifying as male and $54 \%(n=624)$ reported identifying as female. Eighty-nine percent $(n=1,029)$ said they follow politics most or some of the time and 30\% $(n=348)$ were considered highly knowledgeable about politics according to Pew Research Center's (2018) civic knowledge questionnaire. In total, these participants visited 7.5 million websites for our data collection period. YouGov weighted participants to approximate the demographic attributes of the U.S. population and we use these weights in all subsequent calculations and results. Anonymized survey data along with summary web traffic data used for the analyses in the paper are available at: https://osf.io/8fy2z/?view only=0d63bc3fd4f24938867a04efa6084552. Full web traffic histories are not available to protect participant confidentiality. 
Unlike Guess et al. (2020), who only included desktop browsing data in their analysis of exposure to untrustworthy websites in the 2016 election, we chose to include both desktop and mobile browsing data in our analysis of exposure during the 2020 election for two reasons. First, the consumption of news and political information increasingly occurs on mobile devices, and thus it is increasingly important to document mobile exposure to (mis)information (GonzálezBailón \& Xenos, 2022). Approximately 20\% more Americans reported using smartphones each week to access news in 2020 than did in 2016 (Newman et al., 2016, 2020), and nearly twice as many Americans report often getting their news on smartphones than on desktop/laptops (Walker, 2019). Second, a greater proportion of our sample had mobile browsing data available than did Guess et al. (2020)'s sample (the primary reason given by Guess et al. [2020] for not including mobile data in their analyses). In our sample, mobile browsing data was available for $30.1 \%$ of participants, while Guess et al. (2020) only had mobile browsing data for $19 \%$ of their participants.

\section{Measures}

In order to categorize whether a website a participant visited is untrustworthy, we compiled a list of untrustworthy domains in two phases (of course, people can be exposed to misinformation outside of what is measurable in the websites they visit, see the Limitations section for a greater discussion). First, we began with the list of untrustworthy domains used by Guess et al. (2020), which consisted of 490 unique untrustworthy websites collected by previous research. We supplemented this list of 490 websites with 66 additional untrustworthy domains collected by Allcott et al. (2019). Then, we augmented this list of 556 websites with websites from NewsGuard, an organization of former journalists and news editors who manually rate the information quality of websites. We added the 1,240 domains that NewsGuard rated as "repeatedly publishing false content" to the Guess et al. (2020) + Allcott et al. (2019) list of untrustworthy websites, producing a total list of 1,796 unique untrustworthy domains. Next, we matched this list of untrustworthy domains to our participants' URL-level web browsing data to 
identify visits to untrustworthy websites in our sample's web browsing behavior (see the Supplementary Materials for an analysis of our 2020 browsing data limited to the list of 490 untrustworthy websites used in Guess et al. [2020]).

Visits to hard news websites (e.g., www.nytimes.com, www.economist.com) also played a role in Guess et al. (2020)'s analyses. To identify visits to hard news websites in our sample's browsing, we use a database of 500 hard news sites compiled by Bakshy et al. (2015), the same method used by Guess et al. (2020). To ensure that this list of hard news websites is upto-date, we augment this list of 500 sites with all 5,471 websites rated by NewsGuard as not repeatedly publishing false content. 


\section{References}

Alaphilippe, A. (2021, February 4). Disinformation is evolving to move under the radar.

Brookings. https://www.brookings.edu/techstream/disinformation-is-evolving-to-moveunder-the-radar/

Allcott, H., \& Gentzkow, M. (2017). Social Media and Fake News in the 2016 Election. Journal of Economic Perspectives, 31(2), 211-236. https://doi.org/10.1257/jep.31.2.211

Allcott, H., Gentzkow, M., \& Yu, C. (2019). Trends in the diffusion of misinformation on social media. Research \& Politics, 6(2), 2053168019848554. https://doi.org/10.1177/2053168019848554

Allen, J., Howland, B., Mobius, M., Rothschild, D., \& Watts, D. J. (2020). Evaluating the fake news problem at the scale of the information ecosystem. Science Advances, 6(14), eaay3539. https://doi.org/10.1126/sciadv.aay3539

Aslett, K., Guess, A. M., Bonneau, R., Nagler, J., \& Tucker, J. A. (2022). News credibility labels have limited average effects on news diet quality and fail to reduce misperceptions. Science Advances, 8(18), eabl3844. https://doi.org/10.1126/sciadv.abl3844

Auxier, B. (2020, October 15). $64 \%$ of Americans say social media have a mostly negative effect on the way things are going in the U.S. today. Pew Research Center. https://www.pewresearch.org/fact-tank/2020/10/15/64-of-americans-say-social-mediahave-a-mostly-negative-effect-on-the-way-things-are-going-in-the-u-s-today/

Bakshy, E., Messing, S., \& Adamic, L. A. (2015). Exposure to ideologically diverse news and opinion on Facebook. Science, 348(6239), 1130-1132.

https://doi.org/10.1126/science.aaa1160

Ball, P., \& Maxmen, A. (2020). The epic battle against coronavirus misinformation and conspiracy theories. Nature, 581(7809), 371-374. https://doi.org/10.1038/d41586-02001452-z 
Benkler, Y., Faris, R., \& Roberts, H. (2018). Network Propaganda: Manipulation, Disinformation, and Radicalization in American Politics. Oxford University Press. https://doi.org/10.1093/oso/9780190923624.001.0001

Brashier, N. M., \& Schacter, D. L. (2020). Aging in an Era of Fake News. Current Directions in Psychological Science, 29(3), 316-323. https://doi.org/10.1177/0963721420915872

Brodsky, J. E., Brooks, P. J., Scimeca, D., Todorova, R., Galati, P., Batson, M., Grosso, R., Matthews, M., Miller, V., \& Caulfield, M. (2021). Improving college students' factchecking strategies through lateral reading instruction in a general education civics course. Cognitive Research: Principles and Implications, 6(1), 23. https://doi.org/10.1186/s41235-021-00291-4

Delcker, J., Wanat, Z., \& Scott, M. (2020, March 16). The coronavirus fake news pandemic sweeping WhatsApp. POLITICO. https://www.politico.eu/article/the-coronavirus-covid19fake-news-pandemic-sweeping-whatsapp-misinformation/

Edelman. (2021). 2021 Edelman Trust Barometer (p. 58). https://www.edelman.com/trust/2021trust-barometer

Edelson, L., \& McCoy, D. (2021, September 22). How Facebook Hinders Misinformation Research. Scientific American. https://www.scientificamerican.com/article/how-facebookhinders-misinformation-research/

Ellison, N. B., Triệu, P., Schoenebeck, S., Brewer, R., \& Israni, A. (2020). Why We Don't Click: Interrogating the Relationship Between Viewing and Clicking in Social Media Contexts by Exploring the "Non-Click." Journal of Computer-Mediated Communication, 25(6), 402-426. https://doi.org/10.1093/jcmc/zmaa013

Fazio, L. K. (2020). Recognizing the Role of Psychological Science in Improving Online Spaces. Psychological Science in the Public Interest, 21(3), 99-102. https://doi.org/10.1177/1529100620972100

Freelon, D., Marwick, A., \& Kreiss, D. (2020). False equivalencies: Online activism from left to 
right. Science, 369(6508), 1197-1201. https://doi.org/10.1126/science.abb2428

Frenkel, S. (2020, November 23). How Misinformation ‘Superspreaders' Seed False Election Theories. The New York Times. https://www.nytimes.com/2020/11/23/technology/election-misinformation-facebooktwitter.html

Garrett, R. K., \& Bond, R. M. (2021). Conservatives' susceptibility to political misperceptions. Science Advances, 7(23), eabf1234. https://doi.org/10.1126/sciadv.abf1234

Geeng, C., Yee, S., \& Roesner, F. (2020). Fake News on Facebook and Twitter: Investigating How People (Don't) Investigate. Proceedings of the $2020 \mathrm{CHI}$ Conference on Human Factors in Computing Systems, 1-14. https://doi.org/10.1145/3313831.3376784

González-Bailón, S., \& Xenos, M. A. (2022). The blind spots of measuring online news exposure: A comparison of self-reported and observational data in nine countries. Information, Communication \& Society, 0(0), 1-19. https://doi.org/10.1080/1369118X.2022.2072750

Grinberg, N., Joseph, K., Friedland, L., Swire-Thompson, B., \& Lazer, D. (2019). Fake news on Twitter during the 2016 U.S. presidential election. Science, 363(6425), 374-378. https://doi.org/10.1126/science.aau2706

Guess, A. (2015). Fact-checking on Twitter: An examination of campaign 2014 (p. 14). American Press Institute. https://www.americanpressinstitute.org/wpcontent/uploads/2015/04/Project-1B-Guess-updated.pdf

Guess, A., Lyons, B., Montgomery, J. M., Nyhan, B., \& Reifler, J. (2019). Fake news, Facebook ads, and misperceptions: Assessing information quality in the 2018 U.S. midterm election campaign (p. 36).

Guess, A. M. (2015). Measure for Measure: An Experimental Test of Online Political Media Exposure. Political Analysis, 23(1), 59-75. https://doi.org/10.1093/pan/mpu010 Guess, A., Nagler, J., \& Tucker, J. (2019). Less than you think: Prevalence and predictors of 
fake news dissemination on Facebook. Science Advances, 5(1), eaau4586. https://doi.org/10.1126/sciadv.aau4586

Guess, A., Nyhan, B., \& Reifler, J. (2020). Exposure to untrustworthy websites in the 2016 US election. Nature Human Behaviour, 4(5), 472-480. https://doi.org/10.1038/s41562-0200833-x

Ju, A., Jeong, S. H., \& Chyi, H. I. (2014). Will Social Media Save Newspapers? Journalism Practice, 8(1), 1-17. https://doi.org/10.1080/17512786.2013.794022

Jungherr, A., \& Rauchfleisch, A. (2022). Negative downstream effects of disinformation discourse: Evidence from the US. SocArXiv. https://doi.org/10.31235/osf.io/a3rzm

Kauk, J., Kreysa, H., \& Schweinberger, S. R. (2021). Understanding and countering the spread of conspiracy theories in social networks: Evidence from epidemiological models of Twitter data. PLOS ONE, 16(8), e0256179. https://doi.org/10.1371/journal.pone.0256179

King, G., \& Persily, N. (2020). A New Model for Industry-Academic Partnerships. PS: Political Science \& Politics, 53(4), 703-709. https://doi.org/10.1017/S1049096519001021

Konitzer, T., Allen, J., Eckman, S., Howland, B., Mobius, M., Rothschild, D., \& Watts, D. J. (2021). Comparing Estimates of News Consumption from Survey and Passively Collected Behavioral Data. Public Opinion Quarterly, 85(S1), 347-370. https://doi.org/10.1093/poq/nfab023

Kozyreva, A., Lewandowsky, S., \& Hertwig, R. (2020). Citizens Versus the Internet: Confronting Digital Challenges With Cognitive Tools. Psychological Science in the Public Interest, 21(3), 103-156. https://doi.org/10.1177/1529100620946707

Li, J., \& Su, M.-H. (2020). Real Talk About Fake News: Identity Language and Disconnected Networks of the US Public's “Fake News" Discourse on Twitter. Social Media + Society, 6(2), 2056305120916841. https://doi.org/10.1177/2056305120916841

Lim, G., Maynier, E., Scott-Railton, J., Fittarelli, A., Moran, N., \& Deibert, R. (2019). Burned After Reading: Endless Mayfly's Ephemeral Disinformation Campaign (Citizen Lab 
Research Report No. 118). University of Toronto. https://citizenlab.ca/2019/05/burnedafter-reading-endless-mayflys-ephemeral-disinformation-campaign/

Lyons, T. (2018, October 19). New Research Shows Facebook Making Strides Against False News. Meta. https://about.fb.com/news/2018/10/inside-feed-michigan-lemonde/

Lytvynenko, J. (2020, December 6). In 2020, Disinformation Broke The US. BuzzFeed News. https://www.buzzfeednews.com/article/janelytvynenko/disinformation-broke-us

Mitchell, A., \& Jurkowitz, M. (2014, March 13). How Readers Get to News Sites: Social, Search and Direct. Pew Research Center's Journalism Project. https://www.pewresearch.org/journalism/2014/03/13/social-search-direct/

Moore, R. C., \& Hancock, J. T. (2022). A digital media literacy intervention for older adults improves resilience to fake news. Scientific Reports, 12(1), 6008. https://doi.org/10.1038/s41598-022-08437-0

Muise, D. L. (2022). Temporality of political content exposure via smartphone screens: $A$ computational description of rapidity and idiosyncrasy. Stanford University.

Munger, K. (2019). The Limited Value of Non-Replicable Field Experiments in Contexts With Low Temporal Validity. Social Media + Society, 5(3), 2056305119859294. https://doi.org/10.1177/2056305119859294

Munger, K. (2022). Temporal Validity as Meta-Science. Open Science Framework. https://osf.io/https://osf.io/5zfwq

Nekmat, E. (2020). Nudge Effect of Fact-Check Alerts: Source Influence and Media Skepticism on Sharing of News Misinformation in Social Media. Social Media + Society, 6(1), 2056305119897322. https://doi.org/10.1177/2056305119897322

Nelson, J. L., \& Taneja, H. (2018). The small, disloyal fake news audience: The role of audience availability in fake news consumption. New Media \& Society, 20(10), 3720-3737. https://doi.org/10.1177/1461444818758715

Newman, N., Fletcher, R., Levy, D. A. L., \& Nielsen, R. K. (2016). Reuters Institute Digital News 
Report 2016 (р. 124). The Reuters Institute.

https://reutersinstitute.politics.ox.ac.uk/sites/default/files/research/files/Digital\%2520New s\%2520Report\%25202016.pdf

Newman, N., Fletcher, R., Schulz, A., Andı, S., \& Nielsen, R. K. (2020). Reuters Institute Digital News Report 2020 (p. 112). The Reuters Institute.

https://reutersinstitute.politics.ox.ac.uk/sites/default/files/2020-06/DNR_2020_FINAL.pdf

Newton, C. (2020, November 6). Social networks solved their 2016 election problems, but their 2020 problems are bigger. The Verge.

https://www.theverge.com/2020/11/6/21552763/election-2020-tech-social-platformsfacebook-twitter-youtube-misinformation

Pasquetto, I., \& Swire-Thompson, B. (2020). Tackling misinformation: What researchers could do with social media data: Introduction. Harvard Kennedy School Misinformation Review. https://doi.org/10.37016/mr-2020-49

Persily, N. (2021). A Proposal for Researcher Access to Platform Data: The Platform Transparency and Accountability Act. Journal of Online Trust and Safety, 1(1), Article 1. https://doi.org/10.54501/jots.v1i1.22

Pew Research Center, pew. (2018, April 26). 10. Political engagement, knowledge and the midterms. Pew Research Center - U.S. Politics \& Policy. https://www.pewresearch.org/politics/2018/04/26/10-political-engagement-knowledgeand-the-midterms/

Rapp, D. N., \& Salovich, N. A. (2018). Can't We Just Disregard Fake News? The Consequences of Exposure to Inaccurate Information. Policy Insights from the Behavioral and Brain Sciences, 5(2), 232-239. https://doi.org/10.1177/2372732218785193

Reeves, B., Ram, N., Robinson, T. N., Cummings, J. J., Giles, C. L., Pan, J., Chiatti, A., Cho, M., Roehrick, K., Yang, X., Gagneja, A., Brinberg, M., Muise, D., Lu, Y., Luo, M., 
Fitzgerald, A., \& Yeykelis, L. (2019). Screenomics: A Framework to Capture and Analyze Personal Life Experiences and the Ways that Technology Shapes Them. HumanComputer Interaction, O(0), 1-52. https://doi.org/10.1080/07370024.2019.1578652

Reeves, B., Robinson, T., \& Ram, N. (2020). Time for the Human Screenome Project. Nature, 577(7790), 314-317. https://doi.org/10.1038/d41586-020-00032-5

Roth, Y., \& Achuthan, A. (2020, February 4). Building rules in public: Our approach to synthetic \& manipulated media. Twitter. https://blog.twitter.com/en_us/topics/company/2020/newapproach-to-synthetic-and-manipulated-media

The Wall Street Journal. (2021, October 1). The Facebook Files. Wall Street Journal. https://www.wsj.com/articles/the-facebook-files-11631713039

Van Duyn, E., \& Collier, J. (2019). Priming and Fake News: The Effects of Elite Discourse on Evaluations of News Media. Mass Communication and Society, 22(1), 29-48. https://doi.org/10.1080/15205436.2018.1511807

Vijaykumar, S., Jin, Y., Rogerson, D., Lu, X., Sharma, S., Maughan, A., Fadel, B., de Oliveira Costa, M. S., Pagliari, C., \& Morris, D. (2021). How shades of truth and age affect responses to COVID-19 (Mis)information: Randomized survey experiment among WhatsApp users in UK and Brazil. Humanities and Social Sciences Communications, 8(1), 1-12. https://doi.org/10.1057/s41599-021-00752-7

Vu, H. T., \& Saldaña, M. (2021). Chillin' Effects of Fake News: Changes in Practices Related to Accountability and Transparency in American Newsrooms Under the Influence of Misinformation and Accusations Against the News Media. Journalism \& Mass Communication Quarterly, 98(3), 769-789. https://doi.org/10.1177/1077699020984781

Wakefield, J. (2021, March 25). Google, Facebook Twitter grilled in US on fake news. BBC News. https://www.bbc.com/news/technology-56523378

Walker, M. (2019, November 19). Americans favor mobile devices over desktops and laptops for getting news. Pew Research Center. https://www.pewresearch.org/fact- 
tank/2019/11/19/americans-favor-mobile-devices-over-desktops-and-laptops-for-gettingnews/

Walker, M., \& Matsa, K. E. (2021, September 20). News Consumption Across Social Media in 2021. Pew Research Center's Journalism Project. https://www.pewresearch.org/journalism/2021/09/20/news-consumption-across-socialmedia-in-2021/

Weeks, B. E., \& Gil de Zúñiga, H. (2019). What's Next? Six Observations for the Future of Political Misinformation Research. American Behavioral Scientist, 0002764219878236. https://doi.org/10.1177/0002764219878236

Weeks, B. E., Menchen-Trevino, E., Calabrese, C., Casas, A., \& Wojcieszak, M. (2021). Partisan media, untrustworthy news sites, and political misperceptions. New Media \& Society, 14614448211033300. https://doi.org/10.1177/14614448211033300

Wilson, T., \& Starbird, K. (2021). Cross-platform Information Operations: Mobilizing Narratives \&amp; Building Resilience through both "Big" \&amp; "Alt" Tech. Proceedings of the ACM on Human-Computer Interaction, 5(CSCW2), 345:1-345:32. https://doi.org/10.1145/3476086

Wineburg, S., Breakstone, J., McGrew, S., Smith, M. D., \& Ortega, T. (2022). Lateral reading on the open Internet: A district-wide field study in high school government classes. Journal of Educational Psychology, No Pagination Specified-No Pagination Specified. https://doi.org/10.1037/edu0000740

Wurmser, Y. (2019, May 30). US Time Spent with Mobile 2019. Insider Intelligence. https://www.emarketer.com/content/us-time-spent-with-mobile-2019

Zadrozny, B. (2021, July 31). How one man's viral rant shows TikTok's problem with misinformation. NBC News. https://www.nbcnews.com/tech/tech-news/tiktok-audiogives-new-virality-misinformation-rcna1393 


\section{Table 1}

\begin{tabular}{|c|c|c|}
\hline & \multicolumn{2}{|c|}{ Exposure to at least one untrustworthy website (binary) } \\
\hline & 2016 & 2020 \\
\hline Trump supporter & $\begin{array}{c}0.164^{* * *} \\
(0.021)\end{array}$ & $\begin{array}{c}0.138^{* * *} \\
(0.027)\end{array}$ \\
\hline Political knowledge & $\begin{array}{l}0.017^{* *} \\
(0.005)\end{array}$ & $\begin{array}{c}0.049^{* * *} \\
(0.012)\end{array}$ \\
\hline Political interest & $\begin{array}{c}0.048^{* * *} \\
(0.014)\end{array}$ & $\begin{array}{l}0.043^{* *} \\
(0.015)\end{array}$ \\
\hline College & $\begin{array}{c}0.040 \\
(0.022)\end{array}$ & $\begin{array}{c}0.049 \\
(0.028)\end{array}$ \\
\hline Female & $\begin{array}{l}-0.022 \\
(0.020)\end{array}$ & $\begin{array}{c}0.006 \\
(0.026)\end{array}$ \\
\hline Non-white & $\begin{array}{c}-0.094^{* * *} \\
(0.021)\end{array}$ & $\begin{array}{c}-0.071^{*} \\
(0.029)\end{array}$ \\
\hline Age $30-44$ years & $\begin{array}{c}0.041 \\
(0.032)\end{array}$ & $\begin{array}{l}-0.007 \\
(0.041)\end{array}$ \\
\hline Age $45-64$ years & $\begin{array}{l}0.064^{*} \\
(0.029)\end{array}$ & $\begin{array}{c}0.042 \\
(0.038)\end{array}$ \\
\hline Age $65+$ & $\begin{array}{c}0.129^{* * *} \\
(0.033)\end{array}$ & $\begin{array}{l}0.113^{* *} \\
(0.043)\end{array}$ \\
\hline Constant & $\begin{array}{c}0.126^{* *} \\
(0.046)\end{array}$ & $\begin{array}{l}-0.080 \\
(0.059)\end{array}$ \\
\hline Observations & 2,514 & 1,151 \\
\hline $\mathrm{R}^{2}$ & 0.092 & 0.105 \\
\hline Adjusted $\mathrm{R}^{2}$ & 0.089 & 0.098 \\
\hline Residual Std. Error & $0.466(\mathrm{df}=2504)$ & $0.421(\mathrm{df}=1141)$ \\
\hline F Statistic & $28.265^{* * *}(\mathrm{df}=9 ; 2504)$ & $14.827^{* * *}(\mathrm{df}=9 ; 1141)$ \\
\hline
\end{tabular}

Note: OLS regression coefficients are shown with standard errors in parentheses (models estimated using survey weights). The model on the left represents the 2016 election and the model on the right represents the 2020 election. The dependent variable in both models is a binary variable indicating whether an individual was exposed to at least one untrustworthy website during the data collection period (1) or not (0). "Trump supporter" variable: intending to vote for Trump in 2020 election $=1$; not intending to vote for Trump in 2020 election $=0$. "Political knowledge" variable: variable ranging from 0-4 representing the number of questions in Pew Research Center's civic knowledge questionnaire answered correctly out of 4. "Political interest" variable: variable ranging from 1-4 where $4=$ people who say they pay attention to what's going on in government and politics "most of the time" and $1=$ those who pay attention "hardly at all". "College" variable: 1 = college graduate; $0=$ not a college graduate. "Female" variable: $1=$ indicated identifying as a female, $0=$ did not indicate identifying as a female. "Non-white" variable: $1=$ indicated identifying as a race other than white; $0=$ indicated identifying as white. P-values are two-sided. 
Figure 1

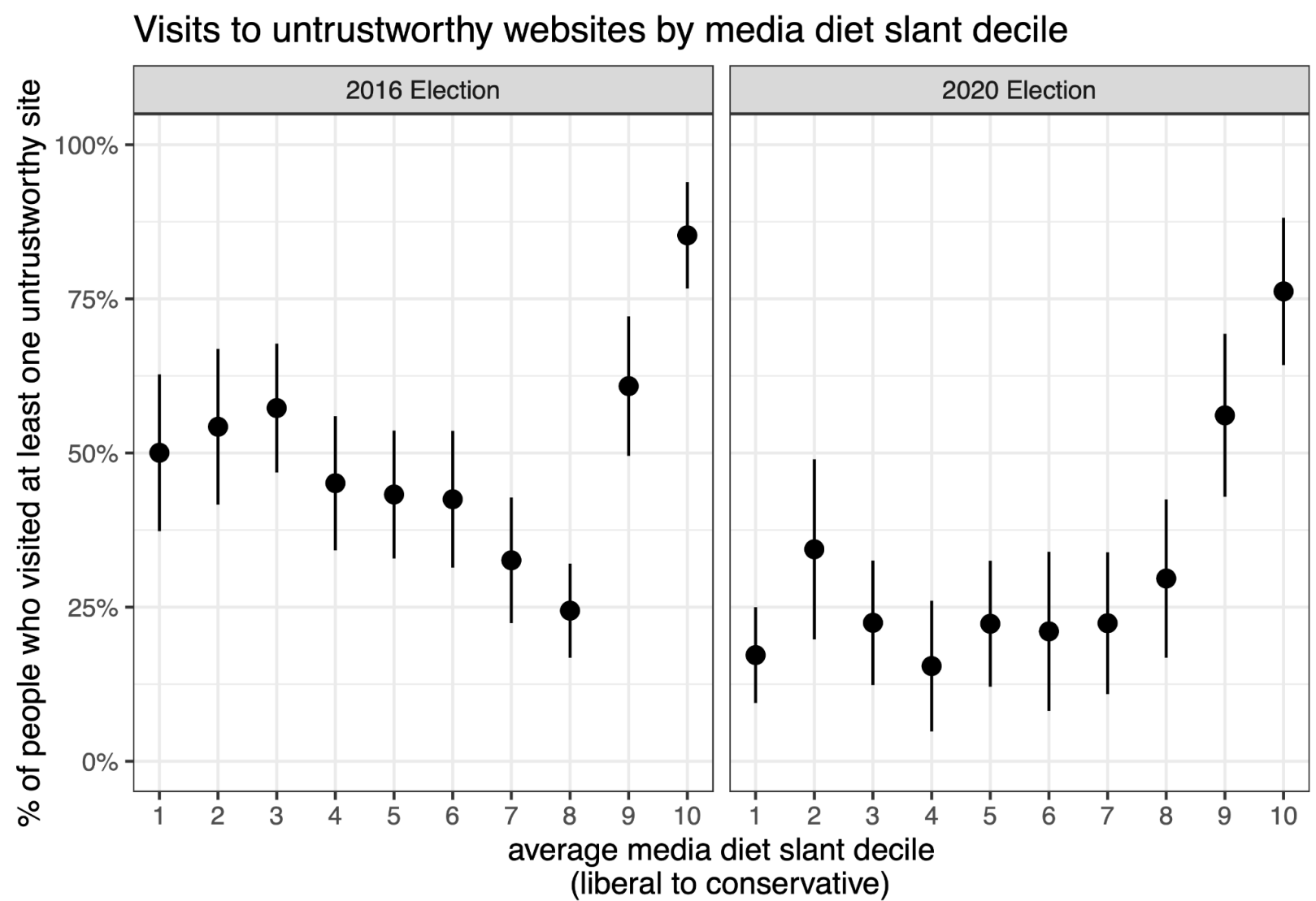

Note: Estimated percentage of people in media diets with different ideological slants who were exposed to at least one untrustworthy website. The panel on the left represents 2016 and the panel on the right represents 2020. Individuals' media diets were split into deciles based on the ideological slant of their news consumption as determined by ideological ratings of hard news websites from Bakshy et al. (2015). Along the x-axis, lower values represent more liberal media diets, middle values represent more moderate media diets, and higher values represent more conservative media diets. Along the y-axis, higher values indicate a greater estimated percentage of people in that media slant decile who were exposed to at least one untrustworthy website. Guess et al. (2020) calculated 2016 deciles separately for Trump and Clinton supporters; we recalculated them to be among all participants using their publicly available replication data. Bars are $95 \%$ confidence intervals. 
Figure 2

\section{Proportion Exposed to Untrustworthy Websites by Age Group}
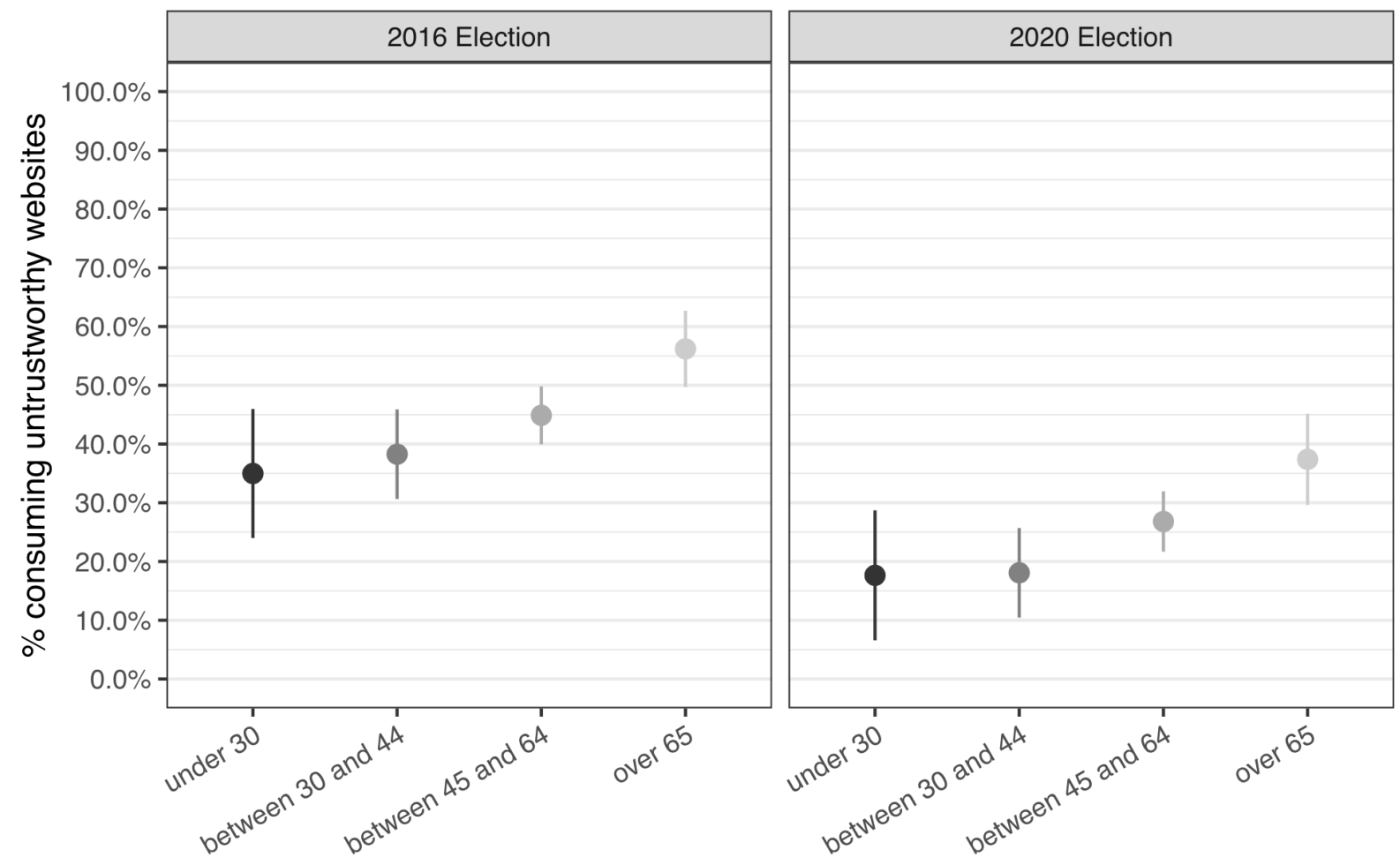

Note: Estimated average percentage of people in different age groups (under 30, between 30 and 44 , between 45 and 64,65 and older) who were exposed to at least one untrustworthy website. The panel on the left represents 2016 and the panel on the right represents 2020. Higher values indicate a greater estimated percentage of people in each age group who were exposed to at least one untrustworthy website. Bars are $95 \%$ confidence intervals. 
Figure 3

\section{Referrers to untrustworthy news websites and other sources}

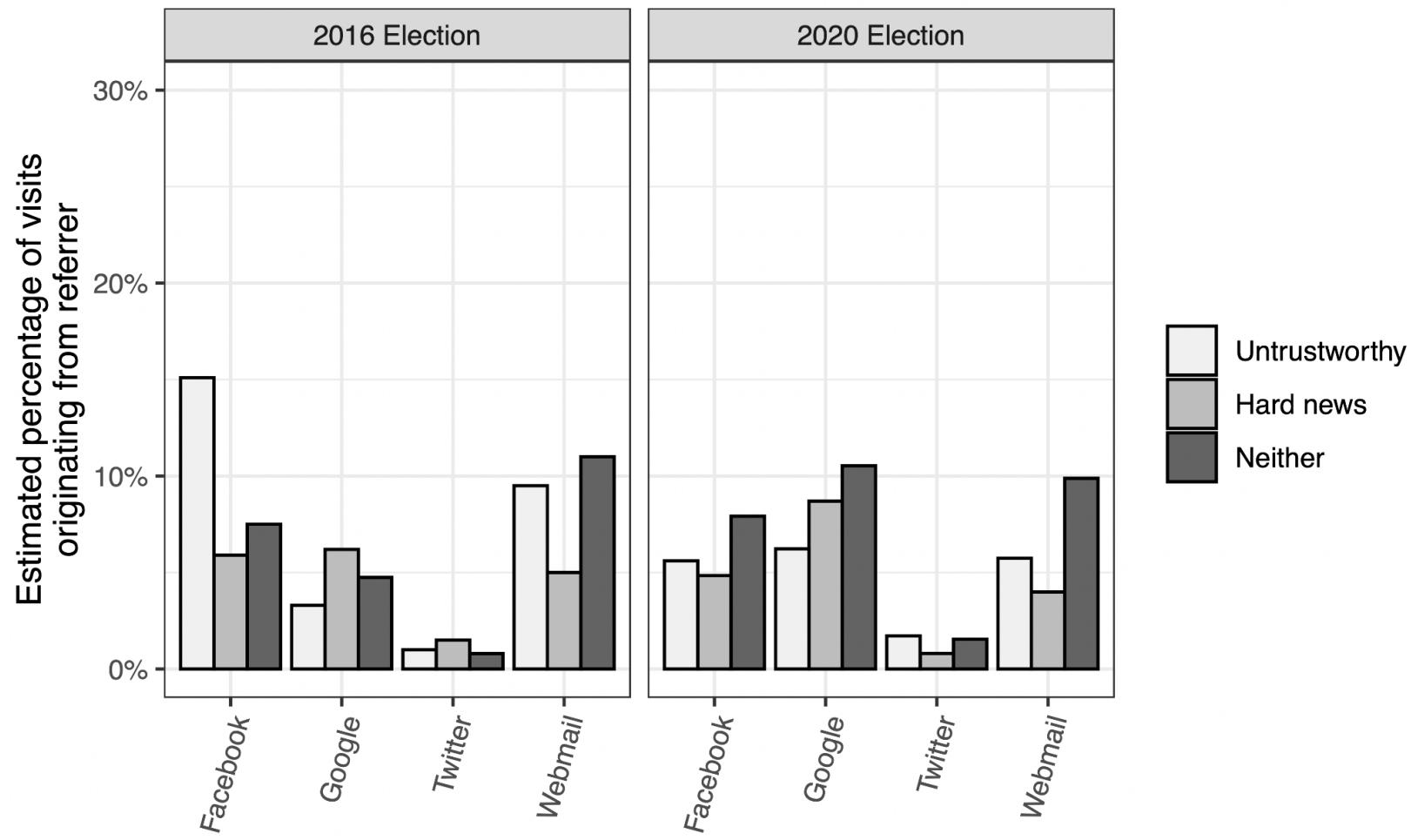

Note: Percentage of total visits to untrustworthy websites, hard news websites, and websites which were neither untrustworthy nor hard news that were referred by different platforms (Facebook, Google, Twitter). The panel on the left represents 2016 and the panel on the right represents 2020. Higher values indicate a greater percentage of visits to each type of website which were preceded by visits to a platform. 